\title{
CGM bessert Kontrolle bei Typ-1-Diabetikern mit eingeschränkter Hypo-Wahrnehmung
}

Hintergrund $u$. Fragestellung: In Studien wurde unter Einsatz der kontinuierlichen Glukosemessung (continuous glucose monitoring; CGM) eine Verbesserung der Glukoseeinstellung dargestellt. Patienten mit einer eingeschränkten Hypoglykämiewahrnehmung schloss man aus diesen Studien aber weitgehend aus. In dieser Studie nun wurde der Effekt von CGM auf die Blutzuckerkontrolle bei Patienten mit einem Diabetes Typ 1 und einer eingeschränkten Hypoglykämiewahrnehmung untersucht.

Patienten und Methoden: In der offenen, randomisierten Crossover-Studie erfolgte bei 52 Patienten mit ICT oder Insu-

T1

Prozentualer Anteil der Zeit, die Patienten in bestimmten Glukosebereichen verbracht haben. (Mittelwert [95\%-KI])

\begin{tabular}{llll}
\hline Glukosebereich & CGM-Phase & SMBG-Phase & p-Wert \\
$4,0-10 \mathrm{mmol} / \mathrm{I}$ & $65,0 \%[62,8-67,3]$ & $55,4[53,1-57,7]$ & $<0,0001$ \\
$\leq 3,9 \mathrm{mmol} / \mathrm{l}$ & $6,8 \%[5,2-8,3]$ & $11,4 \%[9,9-13,0]$ & $<0,0001$ \\
$>10 \mathrm{mmol} / \mathrm{l}$ & $28,2 \%[25,1-31,3]$ & $33,2 \%[30,0-36,3]$ & $<0,0001$ \\
\hline
\end{tabular}

Originalie

van Beers CA, DeVries JH, Kleijer $\mathrm{SJ}$ et al. Continuous glucose monitoring for patients with type 1 diabetes and impaired awareness of hypoglycaemia (IN CONTROL): a randomised, openlabel, crossover trial. Lancet Diabetes Endocrinol. 2016 Nov;4(11):893-902. linpumpe für 6 Wochen eine herkömmliche punktuelle Bestimmung der Glukosekonzentration im Kapillarblut (self measurement of blood glucose; SMBG) oder die Kontrolle mit einem CGM-System (iPro 2 Glukosemonitor und Enlite Glukosesensor; Medtronic; Northridge; USA). Während der

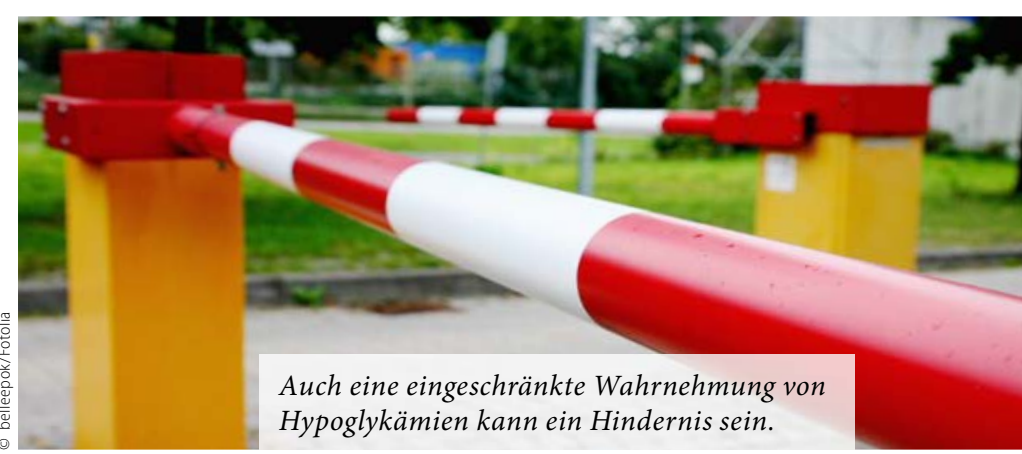

SMBG-Phase wurde das Glukoseprofil mit einem verblindeten CGM-System parallel zur SMBG aufgezeichnet. Es gab darüber hinaus keine Änderung der vorbestehenden Therapie. Primäres Zielkriterium war die Zeit im normoglykämischen Bereich von 4-10,0 mmol/l. Als sekundäre Zielkriterien wurden hypo- und hyperglykämische Episoden erfasst.

Ergebnisse: Die Patienten verbrachten mit CGM mit 65\% (62,867,3; 95\%) signifikant länger im normoglykämischen Bereich als mit SMBG mit 55,4\% (53,1-57,7); $\triangleright$ Tab. 1. Niedrige Werte $\leq 3,9$ mmol wurden durch CGM um 9,8\%, $\leq 3,5 \mathrm{mmol}$ um $25,0 \%$ und Werte $\leq 1,8 \mathrm{mmol}$ um $44 \%$ reduziert. Schwere Hypoglykämien mit Fremdhilfe traten bei CGM 14mal auf, mit SMBG wurden 34 schwere Hypoglykämien mit Fremdhilfe beobachtet. Hyperglykämien mit Glukosewerten $>10 \mathrm{mmol} / \mathrm{l}$ waren mit $28,2 \%$ signifikant seltener als unter SMBG mit 33,2\%.

Schlussfolgerungen: CGM führt bei Typ-1-Diabetes und eingeschränkter Hypoglykämiewahrnehmung zu einer verbesserten Blutzuckerkontrolle und reduziert das Hypoglykämierisiko.

\section{- Kommentar von Prof. Dr. med. Thomas Forst}

\section{Effektive Therapiehilfe bei gestörter Hypoglykämiewahrnehmung}

Bei der kontinuierlichen Glukosemessung gab es viele technische Verbesserungen. In klinischen Studien konnte durch sie eine optimierte Glukosekontrolle mit Reduktion des $\mathrm{HbA}_{1 \mathrm{c}}$ Wertes ohne Anstieg der Hypoglykämiefrequenz belegt werden. In einer Cochrane-Metaanalyse von 22 randomisierten klinischen Studien ergab sich kein Vorteil der CGM bei der Vermeidung von Hypoglykämien. Allerdings wurden in den einbezogenen Studien Patienten mit einem erhöhten Hypoglykämierisiko weitgehend ausgeschlossen. Die nun vorliegende Studie belegt somit erstmalig einen klinischen Nutzen der CGM zur Vermeidung von Unterzuckerungen in einem Kollektiv mit erhöhtem Hypoglykämierisiko. Gerade für Patienten mit eingeschränkter Hypoglykämiewahrnehmung kann die CGM eine entscheidende Therapiehilfe sein.

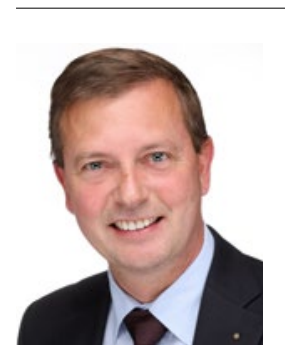

Prof. Dr. med. Thomas Forst

Profil Institut für Stoffwechselforschung $\mathrm{GmbH}$ Hellersbergstr. 9, 41460 Neuss

Thomas.forst@profil.com 